\section{THE DIAGNOSIS AND TREATMENT OF}

$$
\text { E M P Y M A.* }
$$

BY

SIR WILLIAM HALE-WHITE, K.B.E., M.D., CONSULTING PHYSICIAN TO GUY'S hOBPITAL.

Not long ago Dr. H. C. Cameron said he wished that, when next giving a medical address, I would urge the necessity of the early diagnosis of empyema. I do so gladly, for very many instances have come before me in which serious harm has ensued to the patient because an empyema has not been discovered as soon as it ought to have been. This common failing has been the subject of emphatic, strongly worded, bedside teaching. I have often said that the most useful instrument for a consulting general physician is an exploring needle. Some plume themselves on the discovery of an empyema, holding a pint of pus, or one which has caused pyrexia for a fortnight; they should rather feel humiliated because they have not recognised it earlier. Empyema is not a light affair ; it is common, it has a high mortality, and, if not properly treated, the results may be disastrous, even if the patient escapes with his life.

Taking a recent series of 17 consecutive years at Guy's Hospital, the average annual number of cases of empyema is 46.5 in the medical wards alone, and the mortality among these is 29 per cent. No doubt in some, death was not caused by the empyema; the severity of the accompanying pneumonia may have been to blame, or the empyema may have been due to a growth, Bright's disease, or a general septicæmia ; still, in many instances, death was due to the fact that the empyema was not found early enough. Often the patient, before admission to the hospital, already has a large collection of pus in the pleural cavity. Not only do many die, but if the empyema is left a long while the patient is ill for months before he recovers, and even then his lung may be seriously damaged. If we make a post-mortem examination on a patient who has died, without operation, after a large amount of pus has accumulated in the pleural cavity, we find the underlying lung compressed into a carnified mass, with a thick layer of organised lymph over it; such a lung, even had the patient lived, has been rendered for ever useless because of the delay in letting out the pus.

- A Post-Graduate Address given to the North London Medical and Chirurgical Society at the Great Northern Hospital on Oct. 14th, 1925 .
There is, in the Guy's Hospital Museum, a specimen in which the thick covering of such a lung has actually become calcareous. Suppose, however, that the patient, much pus having collected, is operated upon several weeks after the pus began to form, the lung is then bound down so that it cannot re-expand, and the heart is pushed over. There is now a huge, pus-secreting cavity, to try to close which the surgeon either takes away most of the ribs or decorticates the lung. Often the operation fails, and the patient is left with a spinal curvature and a tube in his side. These severe operations never ought to be needed; the pus should have been let out early. I remember saying to a surgeon, "You seem unable to cure longstanding empyemata." He truly replied, "I never ought to be called upon to try." As Osler says, "It is sad to think of the number of lives sacrificed annually." More than 80 years ago Dr. H. M. Hughes wrote :-

" It may be confidently asserted that it is the duty of every individual to employ every rational mode for the purpose of arriving at a correct discrimination of disease ; ... either himself minutely to examine the chest if from habit he is capable of appreciating the importance of the physical signs; or if otherwise, to request the assistance of one who is therein more experienced than himself."

Yet pus is still allowed to collect in the chest week after week.

Clearly, empyema is worth a little study. The causes are many, all are microbic, and by far the commonest causative micro-organism is the pneumococcus. Pneumococcal empyema is nearly always associated with pneumonia, but it may rarely occur without any pulmonary affection. Forty years ago Bristowe's was the best and most popular text-book on medicine ; it is strange that he does not mention pneumonia among the causes of empyema. The tubercle bacillus, by itself, does not cause empyema any more than, by itself, it causes suppurative peritonitis. Tubercle of either pleura or peritoneum leads to the effusion of clear fluid ; if pus is present there is a secondary microbic infection, usually, in the pleural cavity, due to the rupture of a tuberculous pulmonary cavity causing pyopneumothorax.

\section{Diagnosis.}

We now come to the question of vital importance -namely, how to recognise that a patient has an empyema. Happily it is now rare for an empyema to be left so long that it points externally. I have seen only one instance in the last ten years. The older writers, describing this state of affairs, told us that the empyema most often pointed where 
the chest wall was thinnest-namely, at the lower border of the pectoralis major. The signs were distension of the side, œdema of the integuments, bulging of the intercostal spaces, distinctness of the superficial veins, circumscribed redness, and either induration or a fluctuating swelling. After the abscess discharged, the ribs became carious, the patient sank and at length died, worn out and exhausted or carried off by septicæmia; indeed, he may still die thus if operation is too long delayed.

There is no royal road for the detection of an empyema; it is found by exercising care and judgment. Physical signs are of great help : they are those of fluid, but often they are difficult to interpret, for there is a large area of thick lymph over the pleura in the neighbourhood of the collection of pus; local tenderness is frequently a very valuable indication of the exact position of the pus. Empyemata are nearly always between the ribs and the lung, usually at the base and back, but in the case of apical pneumonia they may be found at the top of the chest, and when the patient has lain for some time on his back the collection of pus may be close up against the spine. If the doctor fails to find it, he frequently tries to account for his failure by supposing that the empyema is either between the lobes of the lung, between the lung and diaphragm, or between the pericardium and lung. Such situations are excessively rare; nearly always the empyema is in a usual place, but the doctor has missed it. These exceptionally placed empyemata are very uncommon, difficult to discover, and require expert surgery. I do not propose here to consider them further, but wish to warn you that if you suspect one is present you must not use an exploring needle in the usual way, for, to find the pus, it must be driven through the lung, which is undesirable, and, further, if you discover the pus, you will probably infect the lung as you withdraw the needle. Remember that an empyema may be present on both sides, although it is one of the mysteries of medicine that, in spite of the frequency of double pneumonia, double empyema is rare. I find that out of 781 patients with empyema, it was double in 19 , or 2.4 per cent. Do not expect help from $X$ rays, for commonly the patient is too ill to be examined by them, and often the pneumonic lung and the thick pleural lymph make it very difficult to indicate the position of the pus on an $X$ ray picture. Great care may be necessary to find an empyema. The smallest are some of those which cause a rise of temperature after the crisis of the pneumonia. I remember a boy who had an evening temperature, varying from about $100^{\circ}$ to about $101^{\circ} \mathrm{F}$., for three weeks after his crisis; as a result of very careful examination we discovered pus in the chest with an exploring needle. At the operation the empyema was found to contain only half an ounce of pus, but its evacuation led to the complete recovery of the patient, and in many other instances the empyema has been found to hold quite a small quantity of pus.

\section{The Two Kinds of Empyema.}

There are, as was pointed out by Dr. Camer at the recent discussion at the meeting of the British Medical Association at Bath, two moxifn kinds of empyema, the synpneumonic, in whieh the effusion of pus takes place at the same time a.s the pneumonia ; and the metapneumonic, in whish the pus forms after the pneumonia. In the firgt, general symptoms do not help much, for it is difficult to disentangle those due to the pneumona from those due to the purulent affection of t㠃e pleura; but, speaking generally, the sufferer ols much more ill than he otherwise would be. We are, therefore, chiefly dependent on physical sigäs for the diagnosis of the fluid in the chest. In the second, general symptoms are of great help; tye patient has the crisis so common in pneumonod, all seems well, but in a day or two the evening temperature rises, say, to $100^{\circ}$ or $101^{\circ} \mathrm{F}$., and thenceforth this state of affairs continues withica morning temperature of about $99^{\circ} \mathrm{F}$. The puls, respiration, and leucocytosis, although less than during the pneumonia, do not regain their normal level. Sometimes the apyrexial interval is ondy one day, sometimes it is four or five days, af sometimes there is not strictly an apyrexial intervofl, for the temperature does not fall at the crisis कo normal, but only to nearly normal, and then it begins to rise again. This fall of temperatuges curious, for we believe the pneumonia to beddue to the pneumococcus and the empyema to bedwe to the same micro-organism, and that is responsible for the pyrexia; that being so, it difficult to understand why, although these micmoorganisms are constantly present, there should केe any fall of temperature. Also it is difficult to comprehend why, when the micro-organism causes pneumonia, the daily variations of temperature should not be extreme ; when it causes an empyenfa they may be great. In many cases there is jio apyrexial interval; perhaps in them pus has formed in the pleura from the first, but often therre is no proof of this. There is no sharp bounda $y$ between the group with a well-marked apyrexial interval and those with none. In a third smoll group, the continuation of the pyrexia may be dxe to some complication of the pneumonia-e. pericarditis or other pneumonic infection beyoond the lungs and pleura, pus in the joints existi⿸丆irg as well as the empyema. I call to mind a casse in which one empyema was opened, still the temperature remained raised; then an empyemoa on the other side was drained, pyrexia continueg; then a pneumococcal gluteal abscess was opened, after this the temperature became normal and te patient recovered. Rarely, pneumococcal malip nant endocarditis follows pneumonia, accompanied by empyema, or an empyema is part of a genegal pyæmia, then the patient usually does badly; brit I have notes of two cases of generalised pyæn⿺⿻一𠃋十 following confinement, in which operation for empyema was followed by complete recovexy. 
Any of these conditions will prevent a fall of temperature between the pneumonia and the formation of the empyema. We must also remember that a crisis does not occur in more than half the cases of pneumonia, and when it is not present the temperature falls irregularly and tediously, quite apart from complications. Lastly, I once saw a case in which, although the temperature became quite normal after an attack of pneumonia, physical signs led to the insertion of a needle, and pus was withdrawn, and in another the empyema only caused an evening rise of temperature of one degree.

Still, with all these reservations a study of the temperature chart is most important for the diagnosis of a post-pneumonic empyema, and from it, from the condition of the patient, from the persistent rapidity of pulse, and from the physical signs, we can generally form an opinion as to whether an empyema is present, and if we suspect it we must try to locate it by inserting an exploring needle in the situation indicated by the physical signs.

\section{Treatment.}

Up to the year 1844, when an empyema was successfully diagnosed - often it was not, and many were left until they spontaneously discharged, either externally or through the lung-an incision was made into an intercostal space. This, says Dr. H. M. Hughes, ${ }^{1}$ did not provide efficient drainage, the pleural cavity became converted into a fotid abscess, the secretion of pus increased and became offensive, the comfort of the patient was destroyed, irritative fever and prostration supervened, and the individual sank at an earlier period than if he had been left in the hands of nature without the interference of the surgeon. Hughes urged that putting a small trocar into the chest to withdraw the fluid was easy, practical, and safe, and as a means of diagnosis hardly more annoying to the patient than percussion. $\mathrm{He}$ obtained the surgical aid of Mr. Cock, and they pleaded that the right way to treat an empyema was, after the diagnosis had been confirmed with an exploring cannula, to let the fluid out through a larger cannula and to do this again and again as long as was necessary. They published many successful cases. Subsequently some surgeons took to removing a piece of rib or ribs, but only with the object of letting the chest fall in, and so to help the closure of the pus-secreting cavity. In spite of Hughes' teaching, if the surgeon's object was only to let out the pus, he simply made an incision into an intercostal space. It was Sir Arbuthnot Lane who, in $1882,{ }^{2}$ first taught that a piece of rib should be removed to attain sufficient drainage. He wrote :-

" Now I would suggest that a portion of a rib or ribs be removed at first and the cavity be thoroughly drained from the beginning. ... I have not succeeded in finding an account of any similar treatment."

${ }^{1}$ Guy's Hospital Reports, 1844, pp. 57, 355. ${ }_{2}$ Guy's Hospital Reports, 1882 , xli., 45.
Hughes' and Lane's papers form the first and second landmarks in the story of the treatment of empyema. After Lane's publication, empyemata were treated by excision of rib, with the object of ensuring good drainage, but the mortality of operation, although greatly lessened, remained higher than was expected. Two years ago Cameron and Osgood ${ }^{3}$ showed that the main cause for the high mortality after operation was that synpneumonic cases, which are common in young children under 2 years, almost invariably die after removal of a piece of rib. This is confirmed by many other observers. These cases in young children are often examples of a more generalised infection-e.g., in the fatal cases, suppurative pericarditis or peritonitis are often found-than the pneumonia of older patients, and they are too ill for such an operation as removal of a piece of rib. The right thing for them, as Cameron and Osgood and others have told us, and for synpneumonic cases in older patients, is gently to withdraw as much as possible of the pus by a cannula-several withdrawals may be needed-and later on, if necessary, a piece of rib may be excised; but metapneumonic cases should always be treated, as soon as the pus is discovered, by excision of a piece of rib and free drainage. Thus we see that the correct treatment of empyema is a judicious use of the methods of Hughes and Lane. One point is worth remembering-namely, that if any empyema, for which it has been decided to open the chest by incision, is large, much of the pus should first be drawn off with a cannula. I once saw a surgeon remove a piece of rib for a large metapneumonic empyema, and when the chest was opened the pus gushed out and the patient died instantly, probably because the heart had been too suddenly relieved of the pressure displacing it.

Never forget that the evacuation of the pus is but the first stage of the treatment of an empyema ; the second is the re-expansion of the compressed lung. This is attained best by breathing exercises, which have the additional advantages that they prevent the formation of further adhesions and may even break down slight ones already formed, they restore the normal shape of the chest, they improve the general condition, they diminish breathlessness, and, lastly and most importantly, they greatly aid the discharge of pus. They should be begun the day after the operation with the simple exercise of breathing in through the nose against pressure applied by the hands of the operator on the sides of the lower chest ; later on, when the tube is removed, the body is bent laterally as far as possible so that the uninjured part of the thorax is partially compressed; the operator presses on the damaged side and the patient breathes as deeply as he can. Many other exercises have been devised and are described by C. MacMahon. ${ }^{4}$ I had an unusually large number of 
cases under me during the war, and can speak very highly of the beneficial effect of exercises; they are far superior to the older methods of blowing soap bubbles or blowing water from one Woolfe's bottle to another. When the convalescent stage is reached, send the patient to some hilly place, for walking up hills expands the lungs; neither when performing breathing exercises nor when walking should he ever exhaust himself, but day by day he will be able to do more and more. The completeness of recovery will often be a most pleasant surprise.

Occasionally, for some reason or another, an empyema is not discovered, although it exists. Most usually it is then coughed up through the lung, and many of the patients in whom this happens get quite well. This is remarkable, for the pus burrows through the lung without causing serious damage to it and discharges into a bronchus against gravity. Hepatic abscesses are also a good example of the same thing. I saw a man, sent home from the East because he was discharging pus from a hepatic abscess by coughing. On arrival he was a mere skeleton, weighing only $5 \frac{1}{2}$ st., and he was coughing up over a pint of hepatic pus daily; he was too ill for operation, but under open-air treatment he gained weight, expectoration slowly ceased, and he got quite well; at least 10 gallons of pus must have passed through his lung. Once $I$ had a man $X$ rayed because it had been suggested that he had an aneurysm; the chest was normal, except for a dark line from the top of the right diaphragm to the right bronchus. I mentioned this to him, and he told me he had coughed up a hepatic abscess many years previously, and that he soon got perfectly well; the line no doubt represented the fibrous scar of the abscess track through the lung. I could quote many others I have seen, for 40 per cent. of the hepatic abscesses that discharge spontaneously do so through the lung. Many empyemata that discharge through the lung also do well. Eighty years ago the opinion was that a patient did better if the empyema emptied itself through the lung than he did if the surgeon operated. I could give many examples:-

A boy left Great Ormond-street, where, in spite of every care, they were unable to localise an empyema; soon afterwards he coughed it up and got well.

A young man coughed up pus, wintered at Falmouth, and apparently got quite well; but he had not quite emptied the empyema, for in a few months the expectoration recommenced. Again he went to the sea where he got quite well, and when heard of six years later was indulging in various sports.

An elderly doctor refused operation for an empyema which followed an attack of lobar pneumonia; he coughed up the pus and got quite well.

A man, aged 60, coughed up pus from an empyema following pneumonia; he was sent to the seaside, got quite well, and remained so when heard of two years later.
A feeble old man of 72 had pneumonia, followed by empyema; he coughed up pus and recovered completef.

It would be tedious to give more cases, but my experience is that two-thirds or three-quarteps of the cases of empyemata in which the pus cis coughed up do well without any surgical inte. ference. These patients should live in the open dir day and night, have breathing exercises - the exte of these depending on their condition-be fed plenty of good plain food, and cure appears to be sometimes accelerated by an autogenous vaccins. If the sufferer, who is coughing up pus, is doing wed, make no attempt to locate the pus, for he will continue to do well. Often these are cases in whieh the extent of pleural thickening or the position of the empyema make it very difficult to find ; are further, as the pus is constantly being coughed uq, there is never much at one time in its cavity, and therefore the probability of finding it with a needite is not great.

With regard to the cases in which the pus is being expectorated, but the patient is not doing well, in some the empyema is but part of a generalised infection; in others the patient is an unhealthy subject for any severe illness, and these two groups do badly. But there are some, apast from these two groups, in whom the expectoration is not diminishing, and in whom a hectic tempgrat ture persists in spite of the discharge of pus throg the lung. In both these it is desirable to drain the empyema. If, as already mentioned, it is diffiucut to find the pus, because owing to its expectoration the empyema contains but little, it is a good thing to give morphia to stop the coughing for 12 hours; then the pus collects and the needle may find i

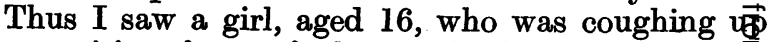
quantities of very foul pus; she had a high hectie temperature and drenching perspirations. might have been expected, we failed to find the empyema, so we gave her morphia in the evening, which made her sleep heavily without coughin? The next morning the empyema was easily found with an exploring needle, drained, and she got quite well. Several times I have obtained hel from morphia in this way.

If the patient has a metapneumonic empyema on both sides, first open one, then aspirate the pis from the other, and when the lung on the side opened has recovered enough drain the oth empyema. For example, a middle-aged man had an empyema on both sides following doubre pneumonia. The usual operation was done on the right side, quantities of pus came away, the lung was much collapsed; a day or two later a pint \& pus was aspirated from the left chest; a wees later the right lung, being still imperfectly expanded, another pint of pus was aspirated from the lef chest; a week later, the right lung being mucd better expanded, an operation was performed of the left chest. The patient did well, and when seen six years after was able to walk at four miles a hour. 